\title{
Lesiones premalignas y malignas del estómago en pacientes sin tamización para cáncer gástrico
}

\author{
Premalignant and malignant findings of the stomach in patients without \\ gastric cancer screening
}

\author{
Hugo Alejandro Bedoya-Arias ${ }^{1} \mathbb{D}$, Carlos Calvache ${ }^{1,2}\left(\mathbb{D}\right.$, Felipe Anduquia ${ }^{1,3}(\mathbb{D}$, \\ Natalia Hurtado ${ }^{1,4} \mathbb{D}$, Santiago Bedoya ${ }^{1,5} \mathbb{D}$, Carlos Ramírez ${ }^{1,6} \mathbb{D}$, Jessica Soto ${ }^{1,7} \mathbb{D}$, \\ Robin Germán Prieto Ortiz ${ }^{1,2} \mathbb{D}$, Julián Orrego ${ }^{1,3} \mathbb{D}$, Bernardo Borráez Segura ${ }^{1,4} \mathbb{D}$
}

Departamento de Ciencias Clínicas, Universidad Tecnológica de Pereira. Colombia.

2 Médico, especialista en Cirugía general, especialista en Gastroenterología y Endoscopia Digestiva, Centro de Enfermedades Hepáticas y Digestivas CEHYD SAS. Bogotá D. C. Colombia.

3 Departamento de Cirugía Gastrointestinal y Endoscopia Digestiva, Liga Contra El Cáncer Risaralda; Universidad Tecnológica de Pereira, Pereira, Colombia.

4 Profesor de Cirugía, Departamento de Ciencias Clínicas, Universidad Tecnológica de Pereira, Pereira, Colombia.

\section{Resumen}

Introducción. Para establecer una frecuencia de seguimiento como método de tamización en cáncer gástrico, se propone la endoscopia en pacientes mayores de 35 años con síntomas de dispepsia, y en pacientes mayores de 40 años con alto riesgo. La demora en la realización de la primera endoscopia en la vida de un paciente incrementa el riesgo de no detectar lesiones premalignas ni cáncer potencialmente prevenible. Los objetivos de nuestro estudio fueron describir el número de pacientes mayores de 40 años con endoscopia de primera vez y evaluar la presencia de lesiones premalignas y malignas del estómago en pacientes sin tamización.

Métodos. Revisión retrospectiva de base de datos. Se describieron hallazgos de informes de histopatología en pacientes mayores de 40 años (lesiones premalignas y malignas). Adicionalmente se describieron las variables sociodemográficas de los pacientes con endoscopia de primera vez y la presencia de infección por Helicobacter pylori en la población mencionada.

Resultados. Setenta y ocho pacientes (23,6 \%) tuvieron una endoscopia de primera vez siendo mayores de 40 años. En el $44 \%$ de los pacientes se encontró la presencia de Helicobacter pylori, 25,4 \% de los pacientes presen-

Fecha de recibido: 19/03/2020 - Fecha de aceptación: 13/05/2020

Correspondencia: Bernardo A. Borráez Segura. Universidad Tecnológica de Pereira. Pereira, Colombia. Calle 22 bis No 4820.

Teléfono: 3102570492

Correo electrónico: bborraez@hotmail.com b.borraez@utp.edu.co

Citar como: Bedoya-Arias HA, Calvache C, Anduquia F, Hurtado N, Bedoya S, Ramírez C, et al. Lesiones premalignas y malignas del estómago en pacientes sin tamización para cáncer gástrico. Rev Colomb Cir. 2020;35:570-4. https://doi.org/10.30944/20117582.583

Este es un artículo de acceso abierto bajo una Licencia Creative Commons - BY-NC-ND https://creativecommons.org/licenses/by-nc$\mathrm{nd} / 4.0 /$ deed.es 
taron atrofia gástrica, 23,I \% metaplasia, ningún paciente presentó displasia y un paciente $(\mathrm{I}, 3 \%)$ presentó un adenocarcinoma gástrico.

Discusión. Los resultados de nuestro estudio muestran un número elevado de pacientes sin endoscopia de tamización. Nuestro estudio resalta la importancia del uso de la endoscopia de tamización en la prevención, así como en el diagnóstico temprano de cáncer gástrico y sugiere mayor adherencia a las guías de práctica clínica.

Palabras clave: endoscopia; cáncer gástrico; tamización; Helicobacter pylori.

\begin{abstract}
Introduction. To establish a frequency of follow-up as a screening method for gastric cancer, endoscopy is proposed in patients older than 35 years with symptoms of dyspepsia, and in patients older than 40 years at high risk. The delay in performing the first endoscopy in a patient's life increases the risk of not detecting premalignant lesions or potentially preventable cancer. The aims of our study were to describe the number of patients older than 40 years with first-time endoscopy and to assess the presence of premalignant and malignant stomach lesions in patients without screening.

Methods. Retrospective database review. Histopathology report findings in patients older than 40 years (premalignant and malignant lesions) were described. Additionally, the sociodemographic variables of patients with first-time endoscopy and the presence of Helicobacter pylori infection in the mentioned population were described.

Results. Seventy-eight patients (23.6\%) had a first-time endoscopy being older than 40 years. Helicobacter pylori was found in $44 \%$ of the patients, $25.4 \%$ of the patients had gastric atrophy, $23.1 \%$ had metaplasia, no patient had dysplasia and one patient (I.3\%) had gastric adenocarcinoma.

Discusions. The results of our study show a high number of patients without screening endoscopy. Our study highlights the importance of the use of screening endoscopy in prevention, as well as in the early diagnosis of gastric cancer, and suggests greater adherence to clinical practice guidelines.
\end{abstract}

Keywords: endoscopy; gastric cancer; screening; Helicobacter pylori.

\section{Introducción}

El cáncer gástrico (CG), es una entidad multifactorial ${ }^{1}$ en la que intervienen diversas causas tanto individuales como ambientales, representadas fundamentalmente por el excesivo consumo de sal, tabaquismo, menor ingestión de verduras y frutas, y el factor principal, la presencia de infección por Helicobacter pylori ${ }^{2}$. Actualmente hay suficiente evidencia de que por lo menos el $90 \%$ de los CG de tipo intestinal, están etiológicamente relacionados con $H$. pylori y que la erradicación de este microorganismo disminuye el riesgo de $\mathrm{CG}^{3,4}$.

En el estadio I del CG, cerca del 6o \% de los pacientes pueden ser asintomáticos, mientras que en los estadios II y III el $26 \%$ y el 2I \% también pueden serlo. Incluso el io \% de los pacientes con estadio IV pueden presentarse sin síntomas, limitando el diagnóstico y una atención adecuada 5 .

En Asia, Europa Oriental, Sur América y América Central, se encuentran los países con mayor incidencia de CG en el mundo. Japón, es uno de los países donde más casos se presentan, pero también en el que se realiza una tamización masiva, En Colombia, donde no hay medidas de tamización obligatorias, es el tercer cáncer más frecuente y el primero en mortalidad. La menor incidencia de CG se observa en África, América del Norte y el Norte de Europa ${ }^{6-8}$. 
La tamización del CG en zonas de alta prevalencia, ha favorecido en gran medida al diagnóstico y el tratamiento temprano, mostrando una reducción significativa en la mortalidad asociada a este ${ }^{6}$. En Colombia, en términos generales la endoscopia está propuesta como el método de tamización en pacientes mayores de 35 años con síntomas de dispepsia y en mayores de 40 años con factores de alto riesgo, con el fin de establecer una frecuencia de seguimiento endoscópico. La demora en la realización de la endoscopia incrementa el riesgo de no detectar a tiempo lesiones premalignas y cáncer potencialmente prevenible?

Los objetivos de nuestro estudio fueron: describir el número de pacientes mayores de 40 años con endoscopia de primera vez y evaluar la presencia de lesiones premalignas y malignas del estómago en pacientes sin tamización.

\section{Métodos}

Se realizó un estudio retrospectivo observacional con muestreo no probabilístico por conveniencia, a pacientes sintomáticos y asintomáticos de la ciudad de Pereira, mayores de I8 años, a quienes se les realizó una endoscopia de vías digestivas altas en un centro de endoscopia, entre julio de 2018 y febrero de 2019. Se excluyeron pacientes menores de 18 años y pacientes con información incompleta en su historia clínica.

Se consideraron los datos sociodemográficos, la presencia de Helicobacter pylori y la identificación de lesiones premalignas y malignas del estómago.

A través del departamento de estadística de la institución, se identificaron 334 pacientes, se recolectó la información de las historias clínicas, informes de endoscopia y de patología mediante el sistema digital institucional y se documentó la información obtenida en una base de datos en Microsoft Excel. Las variables se evaluaron mediante medias aritméticas y porcentajes.

\section{Resultados}

De los 334 pacientes en quienes se realizó una endoscopia digestiva alta, cincuenta y tres pacientes (68\%) fueron mujeres y 25 pacientes (32\%) fueron hombres, con un rango de edad entre 42 y 83 años, con un promedio de 6I,5 años.

La endoscopia se realizó por primera vez en 78 pacientes mayores de 40 años (23,6 \%), de los cuales el $44 \%$ tenía la presencia de Helicobacter pylori, en el 25,4\% de los pacientes se encontró atrofia gástrica, en 23,I \% metaplasia, ningún paciente presentó displasia y en I,3\% de los pacientes (un paciente) se identificó un adenocarcinoma gástrico (tabla I); como hallazgo adicional, el 2,6 \% presentó lesiones premalignas o malignas del esófago: un paciente con esófago de Barrett sin displasia (I,3\%) y uno con diagnóstico de carcinoma escamocelular de esófago $(\mathrm{I}, 3 \%)$.

\section{Discusión}

Los resultados de nuestro estudio muestran que, cerca del $25 \%$ de los pacientes a quienes se les realizó endoscopia en nuestra institución, y por primera vez en la vida, eran mayores de 40 años, con un promedio de edad de 60 años y con un rango entre los 42 y 83 años. De estos pacientes aproximadamente la mitad presentaban Helicobacter pylori, alrededor de un $25 \%$ presentaron atrofia y metaplasia, y solo un paciente presentó cáncer gástrico. Adicionalmente se encontró un paciente con carcinoma escamocelular del esófago.

En Colombia, según GLOBOCAN 20I8, fueron detectados 74I9 Casos nuevos de CG (7,3\% del total de la patología neoplásica) y 5505 muertes debidas a esta enfermedad, representado la primer causa de muerte por cáncer en Colombia ${ }^{8}$, lo que

Tabla 1. Hallazgos premalignos o malignos en estómago, según informes de patología.

\begin{tabular}{lcc}
\hline $\begin{array}{l}\text { Hallazgos premalignos o } \\
\text { malignos en la patología }\end{array}$ & $\mathbf{n}(\%)$ & $\begin{array}{c}\text { Rango de edad } \\
\text { (media) }\end{array}$ \\
\hline Atrofia & $20(25,4)$ & $45-83(63,3)$ \\
Metaplasia completa & $12(15,4)$ & $49-83(63,1)$ \\
Metaplasia incompleta & $6(7,7)$ & $45-77(62,2)$ \\
Displasia & 0 & 0 \\
Adenocarcinoma & $1(1,3)$ & 61 \\
\hline
\end{tabular}


demuestra la importancia de realizar tamización como medida preventiva y con el fin de lograr la detección temprana del cáncer.

El cáncer gástrico es la principal causa de muerte por cáncer en nuestro país, donde frecuentemente se realizan diagnósticos tardíos, es decir en estadios avanzados. la endoscopia en pacientes mayores de 35 años con síntomas de dispepsia y en pacientes mayores de 40 años asintomáticos, permite la detección temprana de lesiones premalignas y malignas, estableciendo además la frecuencia del seguimiento endoscópico.

La realización de la primera endoscopia a mayor edad del paciente, impide la detección temprana de lesiones premalignas o de cáncer potencialmente prevenible ${ }^{6}$. Nuestros resultados muestran que el retraso en la realización de una endoscopia de primera vez, se encuentra en alrededor de 2I, 5 años, identificando en estos pacientes $H$. Pylori en un $44 \%$, con un incremento del riesgo de cáncer de 5,2 a I 8,2 veces dependiendo de su virulencia, según los hallazgos reportados por Holleczek, et al., ${ }^{7}$. Al comparar estos datos con los hallazgos de Lahner y colaboradores, donde se realizaron endoscopias a 979 pacientes entre 50 y 65 años con síntomas de dispepsia, la presencia de $H$. pylori fue mayor en nuestro estudio ( $44 \%$ vs. $34 \%$ ), debido probablemente a la alta incidencia esta infección en nuestro país ${ }^{9}$. Sorpresivamente, este estudio reportó un número mayor de pacientes con atrofia gástrica $(32,9 \%$ vs. $25,4 \%)$ con un riesgo de progresión a cáncer gástrico de o,8 \% según los hallazgos descritos por Den Hoed, et al., pero con presencia de metaplasia en porcentajes similares $(22,9 \%$ vs $23, \mathrm{I} \%$ ) con un riesgo de progresión a cáncer mayor que el de la presencia de atrofia gástrica $(\mathrm{I}, 8 \%)^{\mathrm{Io}}$.

Aunque en este estudio no se encontraron pacientes con displasia, estos pacientes presentan un riesgo de progresión a cáncer que varía de un $4 \%$ a un $33 \%$ considerando que el $25 \%$ de los pacientes con displasias de alto grado pueden progresar a adenocarcinoma en alrededor de I

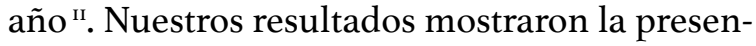
cia de cáncer gástrico en un paciente de 6I años, en quien consideramos que, una endoscopia de tamización a los 40 años con una erradicación adecuada de $H$. pylori o la detección y seguimiento de lesiones premalignas, hubiera modificado el desenlace de este paciente.

\section{Conclusiones}

El presente estudio tiene limitaciones, como el pequeño tamaño de la muestra, pero los resultados indican que, en un número considerable de pacientes, la tamización para cáncer gástrico no se realiza oportunamente, por lo que se recomienda una mayor adherencia a las guías de práctica clínica para lograr la erradicación del H. pylori y la detección temprana de lesiones gástricas premalignas y malignas.

\section{Cumplimiento de normas éticas}

Consentimiento informado: Acorde con la resolución 8430 del Ministerio de Salud, esta investigación se considera de bajo riesgo. Adicionalmente este trabajo contó con la autorización del comité de bioética de la Universidad Tecnológica de Pereira.

La realización de este estudio contó con el aval del comité de bioética de la Universidad Tecnológica de Pereira.

Conflicto de intereses. Los autores declaramos no tener conflicto de interés.

Financiación. Este estudio fue financiado por los autores.

\section{Referencias}

I. Gómez Zuleta MA, Riveros Vega JH, Ruiz O, Concha A, Ángel Betancur DM, Torres Amaya M, et al. Guía de práctica clínica para la prevención, diagnóstico y tratamiento del cáncer gástrico temprano. Rev Colomb Gastroenterol. 2015;30:34-42.

2. Gonzalez CA, Agudo A. Carcinogenesis, prevention and early detection of gastric cancer: where we are and where we should go. Inter J Can. 2012;130:745-53.

3. Gonzalez CA, Sala N, Rokkas T. Gastric cancer: epidemiologic aspects. Helicobacter. 20I3;I8(Suppl I):34-8.

4. de la Riva S, Munoz-Navas M, Sola JJ. Gastric carcinogenesis. Rev Esp Enferm Dig. 2004;96:265-76. 
5. Nakabayashi N, Hirose M, Suzuki R, Suzumiya J, Igawa $M$. How asymptomatic are early cancer patients of five organs based on registry data in Japan. Int J Clin Oncol. 2018;23:999-1006.

https://doi.org/IO.IOO7/sIOI47-0I8-I287-2

6. Oliveros R, Pinilla RE, Facundo Navia H, Sánchez Pedraza R. Cáncer gástrico: una enfermedad prevenible. Estrategias para intervención en la historia natural. Rev Colomb Gastroenterol. 2019;34:177-89.

7. Holleczek B, Schöttker B, Brenner H. Helicobacter pylori infection, chronic atrophic gastritis and risk of stomach and esophagus cancer: Results from the prospective population-based ESTHER cohort study. Int J Cancer. 20I9;I46:2773-83. https://doi.org/IO.I002/ijc.326IO

8. Bray F, Ferlay J, Soerjomataram I, Siegel RL, Torre LA, Jemal A. Global cancer statistics 20I8: GLOBOCAN estimates of incidence and mortality worldwide for 36 cancers in 185 countries. CA Cancer J Clin. 2018;68:394424 .

9. Lahner E, Zullo A, Hassan C, Perri F, Dinis-Ribeiro M, Esposito G, et al. Detection of Gastric Precancerous Conditions in Daily Clinical Practice: A Nationwide Survey. Helicobacter. 20I4;19:417-24. https://doi.org/IO.IIII/hel.I2I49

Io. Den Hoed CM, Holster IL, Capelle LG, de Vries AC, den Hartog B, Ter Borg F, et al. Follow-up of premalignant lesions in patients at risk for progression to gastric cancer. Endoscopy. 2013;45:249-56. https://doi.org/IO.IO55/s-0032-I326379

II. Sung JK. Diagnosis and management of gastric dysplasia. Korean J Inter Med. 2016;31:20I-9. https://doi.org/IO.3904/kjim.20I6.02I 